\title{
Characteristics of low-dimensional plasmons in a metallic strip monolayer on a semiconductor surface
}

\author{
Takeshi Inaoka \\ Department of Materials Science and Technology, Faculty of Engineering, Iwate University, 4-3-5 Ueda, Morioka, Iwate 020-8551, Japan
}

(Received 10 March 2003; published 7 July 2003)

\begin{abstract}
By means of the time-dependent local-density approximation, we investigate low-dimensional plasmons (LDPL's) in a metallic strip monolayer on a semiconductor surface, namely, LDPL's in a two-dimensional conduction-electron system confined in a strip region. We analyze the energy-loss intensity, the energy dispersion, and the induced-charge distribution of the two plasmon modes at each wave number $q$ along the strip. When the wavelength $\lambda(=2 \pi / q)$ of the mode is considerably smaller than the strip width $D$, the higherenergy mode (HEM) has a definite character of the area plasmon (APL), and its energy is close to that of the two-dimensional plasmon in an infinite area (pure 2DPL). However, as the mode energy deviates upward from that of the pure 2DPL with an increase in $\lambda$, the induced-charge distribution of the APL evolves into a standing-wave pattern with its free end at the edge. In contrast, the lower-energy mode (LEM) has a definite character of the edge plasmon. When $\lambda$ is small compared with $D$, the induced charge density of the LEM decays slowly on the inside of the strip owing to the influence of the HEM (APL) close to the LEM in energy.
\end{abstract}

DOI: 10.1103/PhysRevB.68.041301

PACS number(s): 73.21.-b, 73.63.-b, 73.22.-f

The Si (111) $-\sqrt{3} \times \sqrt{3}-\mathrm{Ag}$ surface can be formed by depositing one monolayer of $\mathrm{Ag}$ atoms on a $\mathrm{Si}(111)-7 \times 7$ surface at temperatures higher than $250^{\circ} \mathrm{C} .{ }^{1-3}$ One of the three surface-state bands known, namely, the $S_{1}$-state band provides an ideal two-dimensional (2D) system of conduction electrons. The electron density in this band varies among experiments, ${ }^{4-6}$ because it significantly depends upon presence of a minute amount of extra $\mathrm{Ag}$ adatoms ${ }^{4-6}$ or other possible dopant impurities or surface states.

The conduction-electron character of the $S_{1}$ states can be visualized in low-temperature scanning-tunnelingmicroscopy (STM) images from a $\sqrt{3} \times \sqrt{3}-\mathrm{Ag}$ domain surrounded by atomic steps or out-of-phase (OP) boundaries. ${ }^{2,7}$ One can observe those standing waves which originate from interference of electronic waves impinging on and reflected from the steps or boundaries. Near the step or the OP boundary, we clearly notice corrugation patterns due to this interference with intervals of a few tens of $\AA$, even if the $\sqrt{3}$ $\times \sqrt{3}$ - Ag structure is established on both sides of the step or boundary. ${ }^{7}$ This indicates that the $S_{1}$ electrons are confined in each $\sqrt{3} \times \sqrt{3}-\mathrm{Ag}$ domain by a potential barrier at the step or boundary. The domain can have various shapes, such as a circular one, a squarelike one, and a strip extending on a terrace. $^{2,7}$

Quite recently, by means of high-resolution electron energy-loss spectroscopy (HREELS), Nagao et al. have clearly observed two-dimensional plasmons (2DPL's) due to the $S_{1}$-state band that occur in a virtually infinite area. ${ }^{8}$ Taking account of exchange-correlation (XC) effects, Inaoka et al. have investigated the energy dispersion and the energyloss intensity of the 2DPL in relation to the above experiment. ${ }^{9}$

In a bounded 2D electron system occur edge plasmons (EPL's) localized near the edge and area plasmons (APL's) extending widely on the inside. Especially, studies of the EPL's can be traced back to those of edge magnetoplasmons in a bounded 2D electron system on a liquid-helium surface, ${ }^{10-13}$ and in a superlattice structure of half-planes each of which accommodates conduction electrons. ${ }^{14}$ On the theoretical side, the energy dispersion of the EPL in a half-plane ${ }^{15}$ and a strip ${ }^{16}$ in the absence of a magnetic field has been obtained by a local response scheme and a hydrodynamical one, respectively. We treat the case of no magnetic field.

As for narrow quantum strips with parabolic confining potentials, Schaich et al. have analyzed dipole-forbidden collective excitations in the conduction-electron system, ${ }^{17}$ and Ullrich and Vignale have examined dynamical XC effects on damping of collective excitations. ${ }^{18}$

A finite domain of the $\sqrt{3} \times \sqrt{3}$ - Ag structure newly realizes an ideal 2D conduction-electron system confined in a finite region. This structure is observed to extend as far as an atomic step or an OP boundary that acts as a potential barrier for electrons. ${ }^{2,7}$ In the present work, we consider a strip domain of the $\sqrt{3} \times \sqrt{3}-$ Ag structure with finite width. Using the time-dependent local-density approximation (LDA), ${ }^{19}$ we investigate low-dimensional plasmons (LDPL's) in a 2D conduction-electron system constrained to a strip region.

Here, we explain our theoretical framework. We use the phrase "surface plane" to mean a plane right on a semiinfinite dielectric medium of a dielectric constant $\varepsilon_{S}$. We take the $x$ and $y$ axes in the surface plane. Our model system is composed of conduction electrons in the surface plane, a jellium sheet in the surface plane that has a finite width $D$ in the $x$ direction and that extends infinitely in the $y$ direction, and a confining potential $V_{C}(x)$ owing to an atomic step or an OP boundary. The potential $V_{C}(x)$ is assumed to be flat inside the jellium strip and to rise up parabolically as $\gamma(x$ $\left.-x_{e}\right)^{2}$ just outside the jellium edge at $x=x_{e}$. First, we calculate the ground state of our electron system by the LDA, and then electronic excitations by the time-dependent LDA. We employ an XC potential parametrized by Jonson. ${ }^{20} \mathrm{We}$ calculate the dynamical response of our electron system to two external potentials $U_{s}(x, y, t)=U_{0} \exp [i(q y-\omega t)]$ and 
$U_{a}(x, y, t)=2 U_{0}\left[\left(x-x_{c}\right) / D\right] \exp [i(q y-\omega t)]$ with a constant $U_{0}$ and the strip center $x_{c}$ that oscillate in time with angular frequency $\omega$ and that is periodic in the $y$ direction with wave number $q$. The former potential $U_{s}$ causes symmetric plasmon modes where the induced electron-density distribution is symmetric with respect to the center line at $x=x_{c}$, while $U_{a}$ gives rise to antisymmetric modes where the induced electron-density distribution is antisymmetric with respect to the center line. From the induced electron-density distribution, we can evaluate the energy loss per unit area and time that is involved in the dynamical response. This energy loss is directly proportional to $\left|U_{0}\right|^{2}$, and the proportionality constant is defined as the energy-loss function $F_{L}$.

Here, we mention the parameter values for our calculation. We use the electron density in the neutral condition (or the uniform ion density in the jellium) $n_{0}=3.8 \times 10^{13} \mathrm{~cm}^{-2}$ and the electron effective-mass ratio $m^{*} / m_{0}=0.41$ that have been determined in analyzing 2DPL's in an infinite area by fitting the calculated results with experimental ones. ${ }^{9}$ The symbol $m_{0}$ in the above ratio denotes a free-electron mass. Our $\gamma$ value, $\gamma=20 \mathrm{meV} / \AA^{2}$, is large enough to restrict the penetration of electron states out of the jellium to several angstroms. The width $D$ of the jellium strip, the dielectric constant $\varepsilon_{S}$ of the substrate and a relaxation-rate constant $\eta$ in the susceptibility describing the dynamical response of our electron system are taken to be $D=300 \AA, \varepsilon_{S}=11.5,{ }^{21}$ and $\eta=2 \mathrm{meV}$, respectively. The energy-loss function $F_{L}$ is indicated by dimensionless values when length, energy, and time are measured by the effective Bohr radius $a_{B}^{*}=\left(\varepsilon_{S}\right.$ $+1) \hbar^{2} / 2 m^{*} e^{2}$, the effective Rydberg unit Ry* $=e^{2} /\left(\varepsilon_{S}\right.$ +1) $a_{B}^{*}$, and the time unit $\hbar / \mathrm{Ry}^{*}$.

First, we present the result of our ground-state calculation. Figure 1 shows (a) the effective one-electron potential $V_{\text {eff }}(x)$ and (b) the electron-density distribution $n(x)$. In Fig. 1(a), a longer horizontal bar and a series of shorter ones indicate the Fermi energy $E_{F}$ and bottoms of the occupied subbands, respectively. The effective potential $V_{\text {eff }}$ is measured from its value at the strip center $x_{c}=-150 \AA$. In Fig. 1(b), broken lines represent the uniform ion density $n_{0}$ of the jellium sheet. The origin $x=0$ is adjusted to the jellium edge. The steep potential barrier in Fig. 1(a) leads to a remarkable density oscillation near the jellium edge in Fig. 1(b).

Next, we investigate LDPL's in our system. In this paper, we focus our attention on the symmetric modes, though Fig. 3 involves the antisymmetric modes as well. Figure 2 exhibits the $\omega$ dependence of the energy-loss function $F_{L}$ of the symmetric mode at $q=0.015,0.030$, and $0.050 \AA^{-1}$. We find two major loss peaks at each $q$ value. We refer to the higher (lower)-energy resonance modes at $q=0.050$ and $0.015 \AA^{-1}$ as $H_{1}\left(L_{1}\right)$ and $H_{2}\left(L_{2}\right)$, respectively. The $F_{L}$ value is multiplied by five in the curve for $q=0.015 \AA^{-1}$ to clarify the presence of the mode $\mathrm{H}_{2}$. Figure 3 displays the energy dispersion of the LDPL's (filled squares and circles for the symmetric modes, and open squares and circles for the antisymmetric modes) in comparison with the energy dispersion of 2DPL's in an infinite area (pure 2DPL's) (broken curve). The dispersion of the pure 2DPL's has been calculated in the same time-dependent LDA scheme. The single-particle exci-
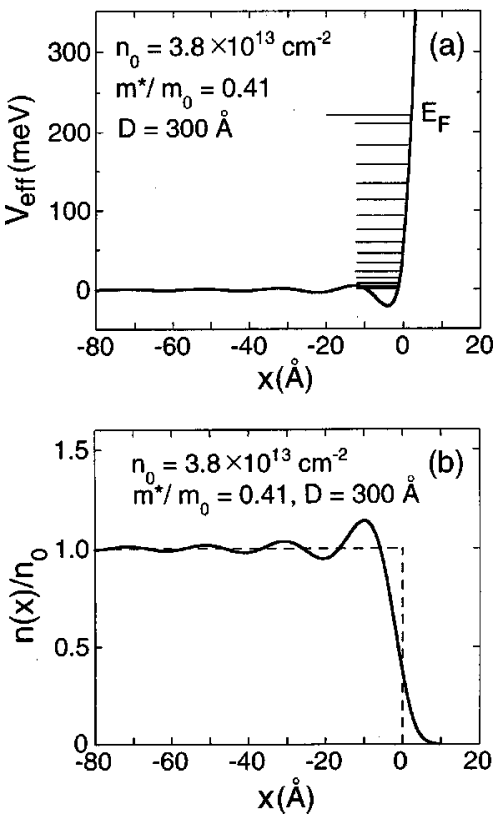

FIG. 1. Ground state of a two-dimensional electron system with density $n_{0}=3.8 \times 10^{13} \mathrm{~cm}^{-2}$ and an effective-mass ratio $\mathrm{m}^{*} / \mathrm{m}_{0}$ $=0.41$ that is confined in a strip region with width $D=300 \AA$. (a) The effective one-electron potential, (b) the electron-density distribution. For details, see the text.

tation (SPE) continuum for the infinite area extends on the right side of the dotted curve. A series of $x$ crosses (plus signs) in a smaller $q$ region has been adapted from the result of the symmetric (antisymmetric) modes by a hydrodynamical scheme in Fig. 2 of Ref. 16. This agrees with our result. The symmetric modes $H_{1}, H_{2}, L_{1}$, and $L_{2}$ in this figure correspond to the resonance peaks in Fig. 2 according to their mode labeling. Figure 4 shows contour maps of the induced electron-density distribution $\delta n$ for the higherenergy modes (HEM's) $H_{1}$ (a) and $H_{2}$ (b) and for the lowerenergy modes (LEM's) $L_{1}$ (c) and $L_{2}$ (d). In each panel of Fig. 4, the position of $x=0 \AA$ and that of $x=-150 \AA$ correspond to the jellium edge and the strip center, respectively. The $y$ axis parallel to the edges is scaled in units of $q^{-1}$. Heavy and light contour lines represent values at regular intervals of opposite signs.

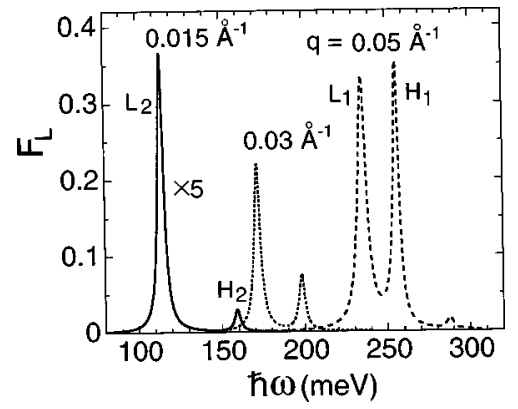

FIG. 2. $\omega$ dependence of the energy-loss function $F_{L}$ of the symmetric plasmon mode at $q=0.015,0.030$, and $0.050 \AA^{-1}$. The $F_{L}$ value is multiplied by five in the curve for $q=0.015 \AA^{-1}$. Four resonance modes marked $H_{1}, H_{2}, L_{1}$, and $L_{2}$ are located in the energy-dispersion diagram of Fig. 3. 


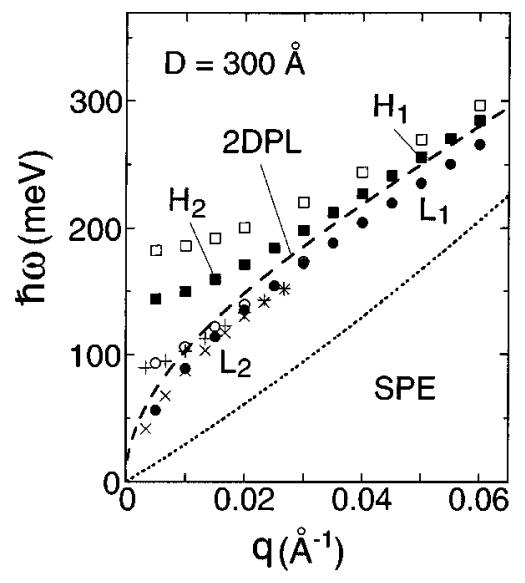

FIG. 3. Energy dispersion of low-dimensional plasmons in a two-dimensional electron system confined in a strip region (filled squares and circles for the symmetric modes, and open squares and circles for the antisymmetric modes). The broken curve shows the energy dispersion of two-dimensional plasmons (2DPL's) in an infinite area. The single-particle excitation (SPE) continuum for the infinite area extends on the right side of the dotted curve. A series of plus signs and $x$ crosses has been adapted from the result of a hydrodynamical calculation in Ref. 16.

In order to understand the behavior of the LDPL's, it is important to note that the wavelength $\lambda(=2 \pi / q)$ becomes equal to the width $D$ at $q \approx 0.02 \AA^{-1}$. Here, we pay attention to the energy dispersion of the symmetric modes in Fig. 3. When $\lambda$ is significantly smaller than $D$, the energy of the HEM (filled squares) is quite close to that of the pure 2DPL (broken curve). With further decrease in $\lambda$, the energy of the HEM approaches that of the pure 2DPL asymptotically. On the other hand, when $\lambda$ becomes so large as to be comparable to $D$, the energy of the HEM begins to deviate upwards from that of the pure 2DPL. This deviation becomes larger with further increase in $\lambda$.

Figure 4(a) exhibits the contour map of $\delta n$ of the mode $H_{1}$ in the small- $\lambda$ regime. The major part of $\delta n$ is found to extend widely inside the strip. This is a definite character of the APL. The induced electron density $\delta n$ has a considerable amplitude around the edge as well, because this mode is close to the LEM (EPL) stated below in energy, and it possesses some share of the character of the EPL. The contour lines flowing from the interior extending part of $\delta n$ down to the edge part of $\delta n$ suggest that the major interior part of $\delta n$ propagates in the $y$ direction dragging the edge part of $\delta n$. Figure 4(b) represents the contour map of $\delta n$ of the HEM $\mathrm{H}_{2}$ with its $\lambda$ value close to the $D$ value. In contrast to the mode $H_{1}$, the induced electron density $\delta n$ has its substantial values both around the strip center $(-150 \AA \leqslant x \lesssim-70 \AA)$ and near the edge $(-50 \AA \lesssim x \leqq 5 \AA)$. The sign of $\delta n$ around the strip center is opposite to that near the edge at each $y$ value. The inside region with substantial $\delta n$ values is restricted to a nearer neighborhood of the strip center in this mode than in the mode $H_{1}$. We can consider that the $\delta n$ distribution in the mode $H_{2}$ forms a standing-wave pattern with its free end at the edge. The contour lines near the edge show remarkable oscillations that result from the interference of electronic
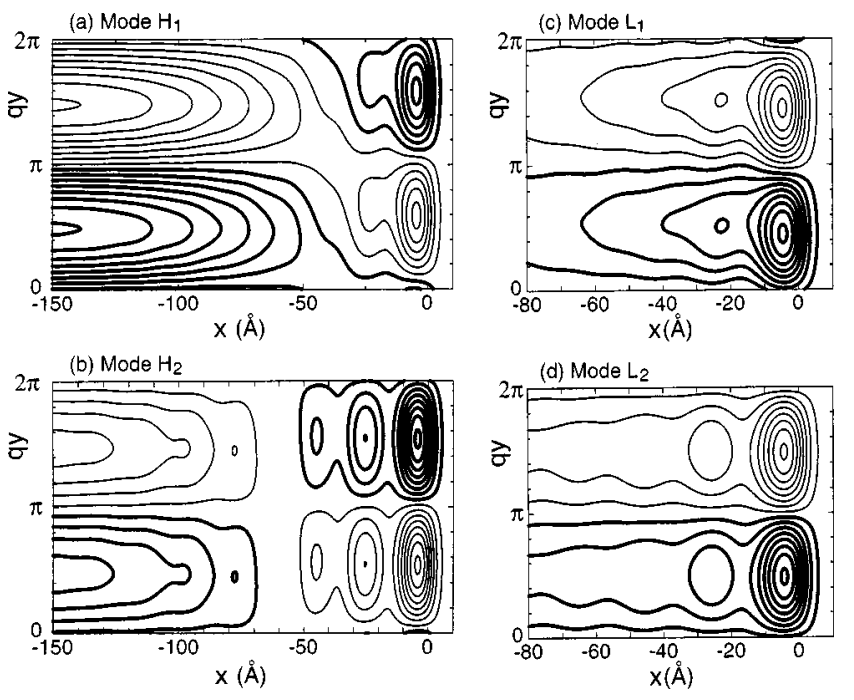

FIG. 4. Contour maps of the induced electron-density distribution of the four symmetric plasmon modes $H_{1}$ (a), $H_{2}$ (b), $L_{1}$ (c), and $L_{2}$ (d) in Fig. 3. Heavy and light contour lines indicate values at regular intervals of opposite signs. The positions of $x=0$ and -150 $\AA$ correspond to the jellium edge and the strip center, respectively.

waves impinging on and reflected from the edge. This oscillation stands out at smaller $q$ values.

Here, we mention the relation of the $\delta n$ distribution and the energy-loss intensity in the HEM. We should recall that $U_{s}$ is constant in the $x$ direction. In the mode $H_{1}$, the interior extending part of $\delta n$ makes a major contribution to the strong energy-loss intensity indicated by the large resonance peak $H_{1}$ in Fig. 2. In the mode $H_{2}$ with the standing-wave pattern, the inside part of $\delta n$ operates to enhance the energyloss intensity, whereas the edge part of $\delta n$ acts to reduce it. This canceling-out relation leads to the very weak energyloss intensity represented by the small resonance peak $H_{2}$ in Fig. 2.

Here, we turn our attention to the LEM. As seen from Fig. 3 , the energy-dispersion curve of the LEM (filled circles) lies somewhat below that of the pure 2DPL. The energy of the LEM comes near that of the HEM, when $\lambda$ is considerably smaller than $D$. On the other hand, as $\lambda$ becomes comparable to $D$, the former energy begins to be separated from the latter energy significantly, and with an increase in $\lambda$, the energy difference becomes larger.

Figures 4(c) and 4(d) exhibit the contour maps of $\delta n$ of the modes $L_{1}$ and $L_{2}$, respectively. What is common to these two modes is that the main structure in the $\delta n$ distribution lies in an edge region $-15 \AA \lesssim x \leqq 5 \AA$ where $n$ rises from zero and builds up a peak near the edge [see Fig. 1(b)]. This is a definite character of the EPL. The main structure in the mode $L_{1}$ with a smaller $\lambda$ value has slowly attenuating tails on the inside, because this mode is close to the HEM (APL) in energy, and acquires something of the extending feature of the APL. On the other hand, the major structure in the mode $L_{2}$ with a larger $\lambda$ value possesses quickly attenuating tails that display conspicuous oscillations due to the 
electronic-wave interference. As exhibited in Fig. 2, with an increase in $\lambda$, the energy-loss intensity of the LEM becomes gradually weaker, but not so quickly as that of the HEM.

We find the same features in the antisymmetric modes. In the HEM's, we recognize the evolution of the inducedcharge distribution from the APL to a standing-wave pattern, while, in the LEM's, we notice the localization near the edges and the slowly attenuating tails on the inside when $\lambda$ $\ll D$. There are remarkable oscillations in the induced-charge density at larger $\lambda$ values in both the HEM's and the LEM's.

Further studies are now in progress to elucidate the dependence of the LDPL's on the strip width and to seek favorable conditions for observing the LDPL's by the HREELS.
${ }^{1}$ S. Hasegawa, X. Tong, S. Takeda, N. Sato, and T. Nagao, Prog. Surf. Sci. 60, 89 (1999).

${ }^{2}$ S. Hasegawa, N. Sato, I. Shiraki, C. L. Petersen, P. Bøggild, T. M. Hansen, T. Nagao, and F. Grey, Jpn. J. Appl. Phys. 39, 3815 (2000).

${ }^{3}$ S. Hasegawa and F. Grey, Surf. Sci. 500, 84 (2002).

${ }^{4}$ Y. Nakajima, S. Takeda, T. Nagao, S. Hasegawa, and X. Tong, Phys. Rev. B 56, 6782 (1997).

${ }^{5}$ R. I. G. Uhrberg, H. M. Zhang, T. Balasubramanian, E. Landemark, and H. W. Yeom, Phys. Rev. B 65, 81305(R) (2002).

${ }^{6}$ J. N. Crain, K. N. Altmann, C. Bromberger, and F. J. Himpsel, Phys. Rev. B 66, 205302 (2002).

${ }^{7}$ N. Sato, S. Takeda, T. Nagao, and S. Hasegawa, Phys. Rev. B 59, 2035 (1999).

${ }^{8}$ T. Nagao, T. Hildebrandt, M. Henzler, and S. Hasegawa, Phys. Rev. Lett. 86, 5747 (2001).

${ }^{9}$ T. Inaoka, T. Nagao, S. Hasegawa, T. Hildebrandt, and M. Henzler, Phys. Rev. B 66, 245320 (2002).
${ }^{10}$ D. B. Mast, A. J. Dahm, and A. L. Fetter, Phys. Rev. Lett. 54, 1706 (1985).

${ }^{11}$ D. C. Glattli, E. Y. Andrei, G. Deville, J. Poitrenaud, and F. I. B. Williams, Phys. Rev. Lett. 54, 1710 (1985).

${ }^{12}$ A. L. Fetter, Phys. Rev. B 32, 7676 (1985).

${ }^{13}$ V. A. Volkov and S. A. Mikhailov, Pis'ma Zh. Eksp. Teor. Fiz. 42, 450 (1985) [JETP Lett. 42, 556 (1985)].

${ }^{14}$ J.-W. Wu, P. Hawrylak, and J. J. Quinn, Phys. Rev. Lett. 55, 879 (1985).

${ }^{15}$ X. Xia and J. J. Quinn, Phys. Rev. B 50, 8032 (1994).

${ }^{16}$ S. Rudin and M. Dyakonov, Phys. Rev. B 55, 4684 (1997).

${ }^{17}$ W. L. Schaich, M. R. Geller, and G. Vignale, Phys. Rev. B 53, 13016 (1996).

${ }^{18}$ C. A. Ullrich and G. Vignale, Phys. Rev. B 58, 7141 (1998).

${ }^{19}$ A. Zangwill and P. Soven, Phys. Rev. A 21, 1561 (1980).

${ }^{20}$ M. Jonson, J. Phys. C 9, 3055 (1976).

${ }^{21}$ H. R. Philipp and H. Ehrenreich, Phys. Rev. 129, 1550 (1963). 